\title{
Supporting the Reuse of Global Unique Identifiers for Individuals in OWL/RDF Knowledge Bases ${ }^{\star}$
}

\author{
Xin Liu ${ }^{1}$, Heiko Stoermer ${ }^{1}$, Paolo Bouquet ${ }^{1}$, and Shu Wang ${ }^{2}$ \\ 1 University of Trento \\ Department of Information Science and Engineering, \\ Trento, Italy \\ \{liu, stoermer, bouquet\}@disi.unitn.it \\ 2 Jilin University \\ Department of Computer Science and Technology, \\ Chang Chun, Ji Lin, China \\ wangshu83@gmail.com
}

\begin{abstract}
In the current state of Semantic Web knowledge representation, it is fairly common that individuals in ontologies have different identifiers even if they are meant to denote the same object in the real world. This makes data integration at the level of individuals quite difficult. In this demonstration, we show two plug-ins called Okkam4N and Okkam4P developed for the NeOn Toolkit and Protégé software respectively, which support the reuse of globally unique identifiers for individuals in OWL/RDF knowledge bases. The key idea is that users are given a chance to look for an existing URI in the publicly available Entity Name System for the corresponding individual in the local OWL/RDF knowledge bases. They are available and tested for the latest official release versions, the NeOn Toolkit 1.2 and Protégé 3.4.
\end{abstract}

\section{Introduction}

One of the major problems that have emerged in the Semantic Web(SW) effort is the problem of uniquely identifying entities [1], or "individuals" on OWL terms. Entities play a major role for the SW since they represent atomic objects of reference and reasoning. Nevertheless, we currently face the problem of uniquely identifying and referencing these entities, which prohibits us from making the next step towards the goal of the SW, that of reasoning about entities in a globally distributed knowledge base. The problem derives from the fact that different users, or systems, assign and use different identifiers for the same realworld entity. As a result, we cannot effectively reason about this entity, exactly because it is not consistently being assigned the same identifier across distributed data sources.

\footnotetext{
* This work is partially supported by the FP7 EU Large-scale Integrating Project OKKAM - Enabling a Web of Entities (contract no. ICT-215032). For more details, visit http://www.okkam.org.
} 
If we could assume that individuals in different ontologies which represent the same actual resources have the same URI, this kind of heterogeneous data problem would be far less significant. In this way, recognizing the same resource distributed in heterogeneous data sources would be only based on simply comparing their identifiers rather than depending on their data structures or content similarities for approximating identity. However, in the current infrastructure of the SW, there is no component to support content creators to reuse already existing URIs of individuals which have already been referred to in other applications or repositories.

The Okkam project 2 is dedicated to solving the issue of identifiers crisis in the SW. It proposes a web-scale infrastructure called the Entity Name System $(E N S)$ 2 to support the reuse of existing URIs for any type of individuals (person, location, organization, event, etc) and provides a few APIs to applications to exchange information with the ENS. The principal mechanism of the ENS is to match the request provided by users or knowledge bases and the entity properties stored in the ENS repository in order to return a list of candidate individuals. This can enable knowledge creation applications to reuse globally unique identifiers for entities.

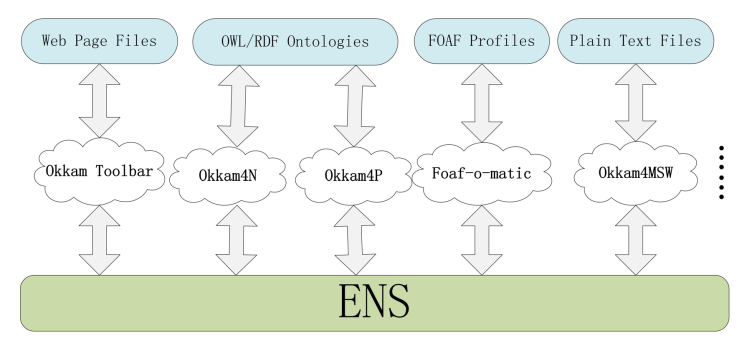

Fig. 1. Currently available ENS-Empowered Tools

But there are various types of content in the SW, html files, RDF documents, relation database management systems, plain text documents and so on. So different ENS-empowered tools 1 are required to aim at different types of knowledge bases. So far, a few client applications have already been developed to deal with entities in some given types of knowledge bases. E.g. Foaf-o-matic [3] is for FOAF profiles; Okkam toolbar is for website contents; Okkam $4 M S W$ is for Microsoft word; the two plug-ins $\operatorname{Okkam}_{4} P\left[4\right.$ and $\operatorname{Okkam}_{4} N$ we present here are for OWL/RDF ontologies. As illustrated in Fig,1.

In this demonstration, we show two plug-ins named Okkam4N and Okkam4P for the NeOn Toolkit2 and Protégé software 3 respectively, as well as their primary

\footnotetext{
${ }^{1}$ ENS-empowered tools are standard end-user applications extended with functionalities which facilitate the creation of okkamized content through the use of ENS. All the currently available tools can be found at http://www.okkam.org/test-tubes

2 http://www.neon-toolkit.org

3 http://protege.stanford.edu
} 
principles and benefits. With the two plug-ins, ontology editors can create ontologies which feature globally unique, shared identifiers for individuals through the use of the ENS. In this way, different knowledge bases can be easily aligned at the level of individuals.

\section{Okkam4N and Okkam4P}

\subsection{Primary Principles}

The Okkam4N uses the Eclips 4 plug-in infrastructure to extend the NeOn Toolkit and the Okkam4P uses the plug-in framework developed at Stanford 5 to extend the functionality for Protégé-OWL editor. Both of them make use of an extension point which allows a plug-in to appear in the right-click popup menu of a selected individual and can be effortlessly hooked into the application architectures to implement the function. The information exchange with the ENS is achieved by invoking the web services provided by the Okkam project.

\section{$2.2 \quad$ Benefits}

In the files generated by the NeOn Toolkit or Protégé, the name of an individual relies on the automatic mechanism of the applictions or the input of human beings. These identifiers are difficult to be understood by other knowledge bases or applications because their scope is usually local. But after being enriched by ENS-empowered tools, the identifiers of various nodes which present the same real-world resource can be assigned to the same globally unique identifier stored in the ENS. So various knowledge bases can be linked together through the nodes which have the same identifier automatically by applications, consequently more useful information will be retrieved from the integrated one.

\section{Demonstration}

We will demonstrate the current versions of Okkam4N and Okkam4P. In order to show the plug-ins more clearly, we present the sequence diagram (Fig 2) to show the process of reusing a globally unique identifier for an individual.

We take the NeOn Toolkit with the Okkam4N plugin and an individual "Barack Obama" with some properties (firstname, lastname, nationality, birthdate, etc) as an example to show how to reuse a pre-existing identifier in local knowledge bases. First of all, users should create and edit the property values of the individual in local applications. Then, in order to reuse a pre-existing identifier for this individual, a request, which consists of the individual type and individual characterism description, will be sent to the ENS. The description is a collection of datatype property name-value pairs and used to distinguish one individual from all the others. Users can get each property from a new popup

\footnotetext{
4 http://www.eclipse.org

5 http://www.stanford.edu/
} 


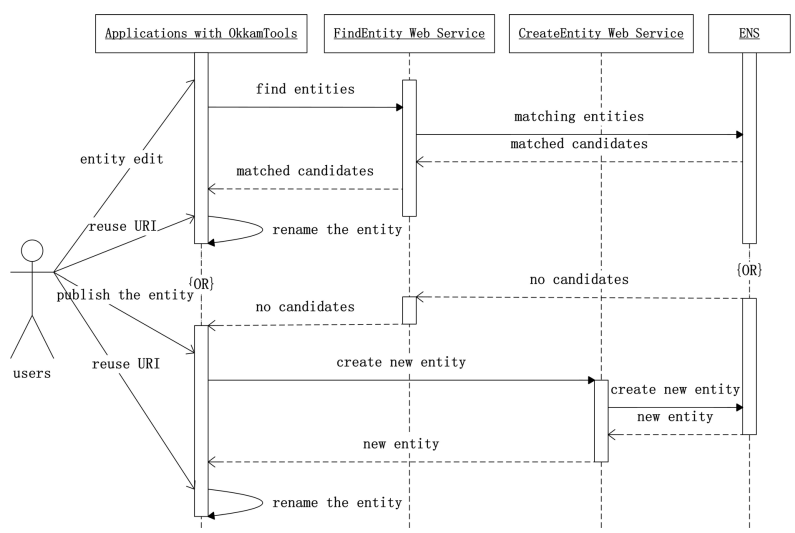

Fig. 2. Sequence diagram of reusing a pre-existing URI

dialog (Fig 3a and assist to generate the request. After looking up the similar individuals, the ENS will return users a ranked list of matched candidates (Fig $3 \mathrm{~b}$ ), among which users can select one correct individual and reuse its identifier, thus causing the substitution of the locally generated identifier with the pre-existing one in the XML code of the ontology; otherwise if no matching individual is found or no matched candidates returned from the ENS, users can choose to publish this individual with its properties to the $E N S$, which will create a new globally unique identifier for it so that other applications or users could reuse it. In this case, the newly created identifier will be used in the XML code.

In this way, various ontology files mentioning "Barack Obama" are interlinked relying on the nodes which share the same identifier, thus generating a semantic environment of this individual (as showed in Fig 4).

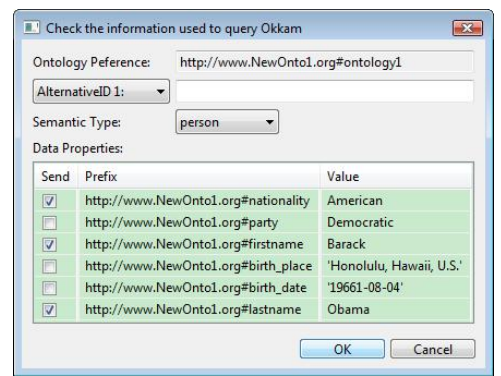

(a) The information of the specific individual

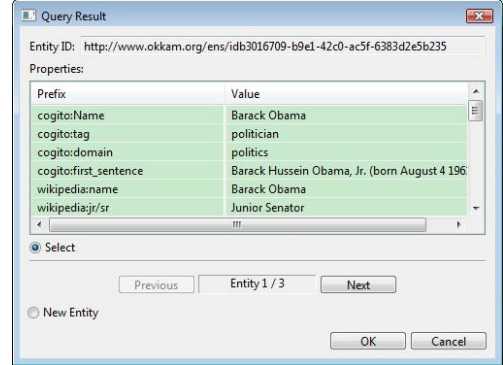

(b) The matched candidate list

Fig. 3. The screensnap of Okkam4N 


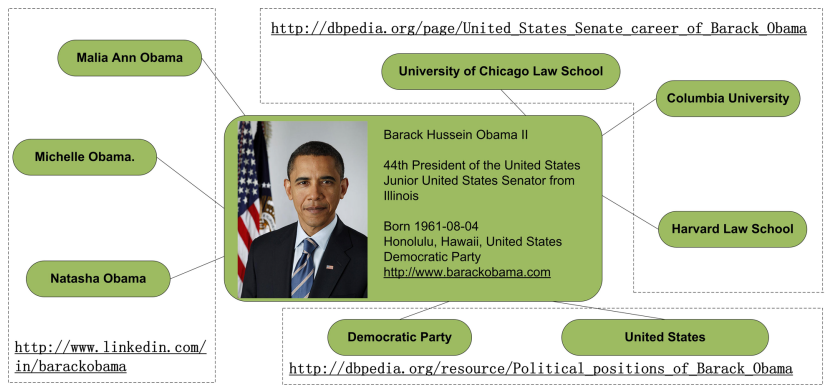

Fig. 4. The semantic environment of Barack Obama

\section{Conclusion}

In this demonstration, we show two plug-ins named Okkam4N and Okkam4P, which can reuse the shared, globally unique identifiers for individuals issued by the ENS in the OWL/RDF knowledge bases created by the NeOn Toolkit or Protégé software.

With the effort of Okkam4N, Okkam4P and other ENS-empowered tools, more useful information would be retrieved from various types of knowledge bases. The diffusion of the use of ENS-empowered tools can help to address the difficulties during the process of data integration.

\section{References}

[1] Bouquet, P., Stoermer, H., Giacomuzzi, D.: OKKAM: Enabling a Web of Entities. In: i3: Identity, Identifiers, Identification. Proceedings of the WWW 2007 Workshop on Entity-Centric Approaches to Information and Knowledge Management on the Web, CEUR Workshop Proceedings, Banff, Canada, May 8 (2007) ISSN 1613-0073, http://CEUR-WS.org/Vol-249/submission_150.pdf

[2] Bouquet, P., Stoermer, H., Niederee, C., Mana, A.: Entity Name System: The Backbone of an Open and Scalable Web of Data. In: Proceedings of the IEEE International Conference on Semantic Computing, ICSC 2008, number CSS-ICSC 2008-4-28-25, pp. 554-561. IEEE Computer Society, Los Alamitos (2008)

[3] Bortoli, S., Stoermer, H., Bouquet, P.: Foaf-O-Matic - Solving the Identity Problem in the FOAF Network. In: Proceedings of the Fourth Italian Semantic Web Workshop (SWAP 2007), Bari, Italy, December 18-20 (2007), http://CEUR-WS.org/Vol-314/43.pdf

[4] Bouquet, P., Stoermer, H., Xin, L.: Okkam4P - A Protégé Plugin for Supporting the Re-use of Globally Unique Identifiers for Individuals in OWL/RDF Knowledge Bases. In: Proceedings of the Fourth Italian Semantic Web Workshop (SWAP 2007), Bari, Italy, December 18-20 (2007), http://CEUR-WS.org/Vol-314/41.pdf 